\title{
Charlson Comorbidity Index Chart Review Version
}

National Cancer Institute

\section{Source}

National Cancer Institute. Charlson Comorbidity Index Chart Review Version. NCI

Thesaurus. Code C106374.

A method of predicting mortality by weighting comorbid conditions calculated by reviewing an individual's chart. 